\title{
Effect of Macitentan on the Pharmacokinetics of the Breast Cancer Resistance Protein Substrates, Rosuvastatin and Riociguat, in Healthy Male Subjects
}

\author{
Dénes Csonka ${ }^{1}\left[\right.$ - Shirin Bruderer ${ }^{1} \cdot$ Armin Schultz $^{2} \cdot$ Marianne Soergel $^{1} \cdot$ Radka Stepanova $^{3} \cdot$ Giancarlo Sabattini $^{4}$. \\ Juan Jose Perez-Ruixo ${ }^{5}$
}

Published online: 24 September 2019

(c) The Author(s) 2019

\begin{abstract}
Background Macitentan is a clinically approved endothelin receptor antagonist for the treatment of pulmonary arterial hypertension (PAH). Increasing use of combination drug therapy in PAH means that it is important to recognize potential drug-drug interactions (DDIs) that could affect the efficacy and safety of macitentan in patients with PAH.

Objective Two Phase 1 studies were conducted to investigate the effect of macitentan at steady-state on the pharmacokinetics of the breast cancer resistance protein (BCRP) substrates, rosuvastatin and riociguat in healthy male subjects. Another objective was to determine the safety and tolerability of concomitant administration of rosuvastatin or riociguat with macitentan. Methods Healthy male subjects received a single oral dose of rosuvastatin $10 \mathrm{mg}(n=20)$ or riociguat $1 \mathrm{mg}(n=20)$ on Day 1 (reference treatment). A loading oral dose of macitentan $30 \mathrm{mg}$ was administered on Day 5 followed by macitentan $10 \mathrm{mg}$ once-daily from Day 6 to Day 15 (riociguat study) or Day 6 to Day 16 (rosuvastatin study). A concomitant oral dose of rosuvastatin $10 \mathrm{mg}$ or riociguat $1 \mathrm{mg}$ was administered on Day 10 (test treatment). Pharmacokinetics were evaluated for $96 \mathrm{~h}$ after treatment on Day 1 and for $144 \mathrm{~h}$ (riociguat study) or $168 \mathrm{~h}$ (rosuvastatin study) after treatment on Day 10 . To compare the reference and test treatments, the geometric mean ratio was calculated for the maximum plasma concentration $\left(C_{\max }\right)$, the area under the plasma concentration-time curve (AUC) from zero (pre-dose) to time of the last measured concentration above the limit of quantification $\left(\mathrm{AUC}_{0-t}\right)$, the $\mathrm{AUC}$ from zero to infinity $\left(\mathrm{AUC}_{0-\infty}\right)$ and the terminal elimination half-life $\left(t_{1 / 2}\right)$ of rosuvastatin, riociguat and riociguat's metabolite, M1. The difference in the time to reach maximum plasma concentration $\left(t_{\max }\right)$ was determined by the Wilcoxon test. Trough levels of macitentan and its metabolite, ACT-132577, were measured and safety was monitored throughout.

Results Ninety percent confidence intervals of the geometric mean ratios were within the bioequivalence criteria of 0.80 1.25. There was no significant difference between test and reference $t_{\max }$. Rosuvastatin or riociguat did not affect the steadystate concentrations of macitentan and ACT-132577. The adverse event profile was consistent with the known safety profiles of the drugs.
\end{abstract}

Conclusions Macitentan $10 \mathrm{mg}$ did not affect the pharmacokinetics of BCRP substrates, rosuvastatin or riociguat in healthy male subjects.

EudraCT numbers: 2017-003095-31 and 2017-003502-41.

Dénes Csonka

dcsonka@its.jnj.com

1 Actelion Pharmaceuticals Ltd, Gewerbestrasse 16, 4123 Allschwil, Switzerland

2 Clinical Research Services Mannheim GmbH, Mannheim, Germany

3 Aixial s.r.o., Brno, Czech Republic

4 Idorsia Pharmaceuticals Ltd., Allschwil, Switzerland

5 Janssen Global Clinical Pharmacology, Turnhoutseweg, Beerse, Belgium

\section{Introduction}

Pulmonary arterial hypertension (PAH) is a progressive disorder in which abnormal smooth muscle proliferation and excess vasoconstriction result in elevated pulmonary arterial pressure [1]. This is caused by an imbalance in vasoactive substances released by the pulmonary vascular endothelium. Of particular interest is the potent vasoactive peptide, endothelin-1 (ET-1), which has been found in increased quantities in the plasma and endothelium of patients with 


\section{Key Points}

Oral administration of macitentan $10 \mathrm{mg}$ had no effect on the pharmacokinetic profile of orally administered breast cancer resistance protein substrates, rosuvastatin and riociguat, in healthy male subjects.

Oral administration of rosuvastatin or riociguat did not affect the steady-state concentrations of macitentan and its active metabolite, ACT-132577.

Concomitant administration of macitentan with rosuvastatin or riociguat was well tolerated and the treatmentemergent adverse event profiles were consistent with the known safety profiles of the drugs.

PAH $[2,3]$. ET-1 exerts vasoconstrictor and vasodilatory effects by binding to endothelin receptor (ETR)-A and ETRB, respectively [4-6].

Combination drug therapy has become increasingly prevalent in the treatment of $\mathrm{PAH}$, and evidence shows that dual combination of PAH-specific therapy delays disease progression in patients [7]. In a registry to evaluate early and long-term PAH disease (REVEAL), $46 \%$ of patients were receiving two and $9 \%$ of patients were receiving three $\mathrm{PAH}-$ specific therapies [8]. Therefore, it is important to recognize potential drug-drug interactions (DDIs) that could affect the efficacy and safety of PAH patient treatment.

Macitentan and its active metabolite, ACT-132577, are dual endothelin receptor antagonists (ERAs) that prevent the binding of ET-1 to ETR-A and ETR-B. Macitentan has a high affinity for the ETRs, with a 50-fold increased selectivity for ETR-A compared to ETR-B [9]. It also has the advantage of having significantly slower dissociation kinetics than other ERAs [10]. Macitentan is approved for the treatment of patients with PAH in the European Union, Canada and the USA. The DDI potential of the approved clinical dose of macitentan $(10 \mathrm{mg})$ is considered to be low and various studies have shown that the pharmacokinetics of macitentan are not affected by commonly prescribed medicines for PAH [11]. More than $99 \%$ of macitentan and ACT-132577 are highly bound to plasma proteins [12]. In vitro, macitentan has been shown to inhibit breast cancer resistance protein (BCRP) transporters with a 50\% inhibitory concentration of $1.0 \mu \mathrm{M}$. BCRP is an efflux pump located in the gut, liver canalicular membrane, and kidney, and is exposed to intracellular drug concentrations in the liver and the kidney. When systemic plasma concentrations of macitentan are corrected for the high plasma protein binding, the resulting unbound plasma concentration is not expected to lead to inhibition of BCRP in the liver or the kidney (unpublished data on file, Actelion Pharmaceuticals Ltd., Allschwil,
Switzerland). However, the extent of the effect, if any, that macitentan has on intestinal BCRP is unknown. Inhibition of BCRP at the intestinal level might result in an increase in the rate, and extent of absorption of BCRP substrates, like riociguat and rosuvastatin.

Our aim, therefore, was to investigate the effect of a clinical dose of macitentan $10 \mathrm{mg}$ on BCRP using two BCRP substrates, rosuvastatin [13] and riociguat [14]. Riociguat is also indicated in the treatment of PAH and is currently the only approved therapy for chronic thromboembolic pulmonary hypertension (CTEPH); it may be administered concomitantly with macitentan [15]. We conducted two Phase 1 DDI studies with the primary objective of evaluating the effect of macitentan at steady-state on the pharmacokinetics of rosuvastatin and riociguat in healthy male subjects. The secondary objectives were to evaluate the effect of macitentan at steady-state on the pharmacokinetics of riociguat's major active metabolite, M1; to evaluate the effect of rosuvastatin and riociguat on the steady-state concentrations of macitentan and its active metabolite, ACT-132577; and to determine the safety and tolerability of concomitant administration of rosuvastatin or riociguat with macitentan.

\section{Methods}

\subsection{Study Design}

An independent ethics committee (Ethik-Kommission bei der Landesärztekammer, Baden-Württemberg, Jahnstrasse 40, 70573 Stuttgart, Germany) and the national health authority of Germany approved the two study protocols. Informed consent was obtained from all study participants. The studies were conducted at Clinical Research Services, Mannheim, Germany in accordance with the Declaration of Helsinki. Both were prospective, open-label, one sequence, two-treatment, Phase 1 studies. The first study was conducted between November 2017 and December 2017 and examined the effect of macitentan at steady-state on the pharmacokinetics of rosuvastatin after a single dose of rosuvastatin (EudraCT number: 2017-003095-31). The second study was conducted between December 2017 and February 2018 and examined the effect of macitentan at steadystate on the pharmacokinetics of riociguat and the riociguat metabolite, M1, after a single dose of riociguat (EudraCT number: 2017-003502-41).

\subsection{Study Subjects}

The studies enrolled healthy, adult males aged $18-55$ years (rosuvastatin study) and 18-45 years (riociguat study). Subjects were required to have a body mass index of $18-30 \mathrm{~kg} / \mathrm{m}^{2}$, normal systolic and diastolic blood pressure and a 
normal 12-lead electrocardiogram (ECG). Exclusion criteria included, but were not limited to, known allergic reactions to the study drugs, excipients of the study drugs, or any drug of the same class; any previous exposure to the study drugs (rosuvastatin study) or previous exposure to study drugs within 3 months prior to screening (riociguat study); and a history of any disease or condition that could interfere with the absorption, distribution, metabolism or excretion of the study drugs. Subjects with levels of hepatic aminotransferases, hemoglobin or hematocrit outside the normal range were also excluded. Subjects of Asian race were excluded from the rosuvastatin study because of the differences in the metabolism of statins in Asians compared with Caucasians [16].

\subsection{Study Drug}

Macitentan was provided by Actelion Pharmaceuticals Ltd. as film-coated tablets for oral administration, formulated at a strength of $10 \mathrm{mg}$ [12]. The same batch of macitentan was administered to all subjects. Commercially available rosuvastatin (10 mg tablets for oral administration) (Crestor AstraZeneca, GmbH, Wedel, Germany) [17] and riociguat (1 mg tablets for oral administration) (Adempas, Bayer Pharmaceuticals AG, Berlin, Germany) [18] were purchased for use in the studies. The same batch of rosuvastatin or riociguat was used for all subjects.

\subsection{Dosing Schedule}

Subjects received a $10 \mathrm{mg}$ single oral dose of rosuvastatin or a $1 \mathrm{mg}$ single oral dose of riociguat on Day 1 (Fig. 1). A $30 \mathrm{mg}$ (three $10 \mathrm{mg}$ tablets) single oral loading dose of macitentan was administered on Day 5, followed by once-daily $10 \mathrm{mg}$ oral doses of macitentan from Day 6 to Day 15 (riociguat study) or Day 6 to Day 16 (rosuvastatin study). On Day 10, a $10 \mathrm{mg}$ single oral dose of rosuvastatin or a $1 \mathrm{mg}$ single oral dose of riociguat was co-administered with macitentan. The subjects were in a fasted state at drug administration on Day 1, Day 5 and Day 10. Study drugs were administered with water. The intake of fluids was not permitted from $1 \mathrm{~h}$ before, until $1 \mathrm{~h}$ after, drug administration on Day 1 and Day 10.

\subsection{Pharmacokinetic Sampling}

Blood samples were collected for rosuvastatin $(5.5 \mathrm{~mL})$, riociguat and $\mathrm{M} 1(4.9 \mathrm{~mL})$ and macitentan and ACT-132577 $(2.7 \mathrm{~mL})$ for pharmacokinetic analysis at the times specified in Table 1. Blood samples were collected for macitentan and ACT-132577 trough concentration $\left(C_{\text {trough }}\right)$ before drug administration and once-daily on Day 5 to Day 16. In the rosuvastatin study, an additional sample was collected on Day 17. Plasma was separated by centrifugation. In the rosuvastatin study, the plasma was mixed with $0.1 \mathrm{M}$ sodium acetate buffer ( $\mathrm{pH}$ 4.0) to prevent the possible conversion of rosuvastatin $5 \mathrm{~S}-$ lactone to rosuvastatin.

\subsection{Bioanalysis}

Validated liquid chromatography with tandem mass spectrometry (MS/MS) methods [19-22] were used for the quantification of rosuvastatin, riociguat, M1, macitentan and ACT-132577 in human plasma samples using their respective stable isotope labelled internal standards. The concentration ranges of the assays were $0.04-25 \mathrm{ng} / \mathrm{mL}$ for rosuvastatin, $0.2-100 \mathrm{ng} / \mathrm{mL}$ for riociguat and $\mathrm{M} 1$ and $1-2000$ $\mathrm{ng} / \mathrm{mL}$ for macitentan and ACT-132577.
Fig. 1 Study design and dosing schedule of the rosuvastatin and riociguat studies

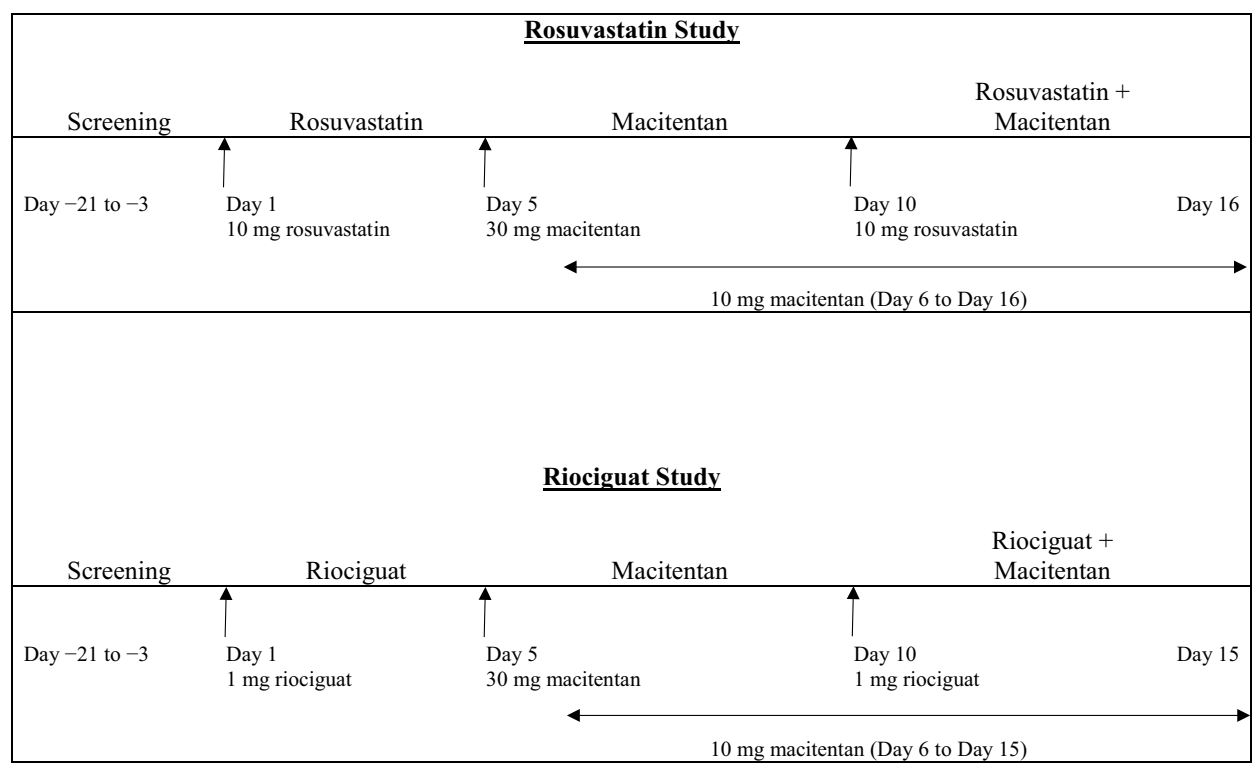


Table 1 Blood sample collection times for rosuvastatin, riociguat and M1 pharmacokinetic analysis

\begin{tabular}{|c|c|c|c|c|c|c|c|c|c|c|c|c|c|c|c|c|c|c|c|c|c|c|c|c|c|}
\hline \multirow{2}{*}{$\begin{array}{l}\text { Study Day } \\
\text { Hours post-dose }\end{array}$} & \multirow{2}{*}{$\begin{array}{l}-1 \\
\text { Pre-dose }\end{array}$} & \multicolumn{19}{|l|}{1} & \multirow[b]{2}{*}{16} & \multirow{2}{*}{$\begin{array}{l}2 \\
24\end{array}$} & \multirow{2}{*}{$\begin{array}{l}3 \\
48\end{array}$} & \multirow{2}{*}{$\begin{array}{l}4 \\
72\end{array}$} & \multirow{2}{*}{$\begin{array}{l}5 \\
96\end{array}$} \\
\hline & & 0.5 & & 1 & 1.5 & 2 & 2.5 & 3 & 3.5 & 4 & & 4.5 & 5 & 5.5 & 6 & 7 & 7 & 8 & 10 & 12 & & & & & \\
\hline Rosuvastatin & $\mathrm{X}$ & $\mathrm{X}$ & & $\mathrm{X}$ & $X$ & $X$ & $X$ & $\mathrm{X}$ & $\mathrm{X}$ & $\mathrm{X}$ & & $\mathrm{X}$ & $\mathrm{X}$ & $X$ & $x$ & $x$ & $X$ & $\mathrm{X}$ & $\mathrm{X}$ & $X$ & $\mathrm{X}$ & $\mathrm{X}$ & $\mathrm{X}$ & $\mathrm{X}$ & $\mathrm{X}$ \\
\hline Riociguat/M1 & $\mathrm{X}$ & $\mathrm{X}$ & & $\mathrm{X}$ & $X$ & $\mathrm{X}$ & $\mathrm{X}$ & $\mathrm{X}$ & - & $\mathrm{X}$ & & - & $X$ & - & $X$ & $x$ & - & $\mathrm{X}$ & $X$ & $X$ & $\mathrm{X}$ & $\mathrm{X}$ & $X$ & $\mathrm{X}$ & $\mathrm{X}$ \\
\hline Study Day & 10 & & & & & & & & & & & & & & & & & & 11 & 12 & 13 & 14 & 15 & 16 & 17 \\
\hline Hours post-dose & Pre-dose & 0.5 & 1 & 1.5 & 2 & 2.5 & 3 & 3.5 & 4 & 4.5 & 5 & 5.5 & 6 & 7 & 8 & 10 & 12 & 16 & 24 & 48 & 72 & 96 & 120 & 144 & 168 \\
\hline Rosuvastatin & $\mathrm{X}$ & $\mathrm{X}$ & $\mathrm{X}$ & $\mathrm{X}$ & $\mathrm{X}$ & $\mathrm{X}$ & $X$ & $\mathrm{X}$ & $\mathrm{X}$ & $\mathrm{X}$ & $\mathrm{X}$ & $\mathrm{X}$ & $\mathrm{X}$ & $\mathrm{X}$ & $X$ & $\mathrm{X}$ & $\mathrm{X}$ & $\mathrm{X}$ & $\mathrm{X}$ & $\mathrm{X}$ & $\mathrm{X}$ & $\mathrm{X}$ & $\mathrm{X}$ & $\mathrm{X}$ & $\mathrm{X}$ \\
\hline Riociguat/M1 & $X$ & $\mathrm{X}$ & $X$ & $\mathrm{X}$ & $X$ & $\mathrm{X}$ & $X$ & - & $X$ & - & $X$ & - & $X$ & - & $X$ & $X$ & $X$ & $X$ & $X$ & $X$ & $X$ & $X$ & $\mathrm{X}$ & $X$ & - \\
\hline
\end{tabular}

$X$ blood sample collected, - blood sample not collected

Rosuvastatin was extracted from acidified plasma samples using liquid-liquid extraction. After evaporation of the organic solvent, the reconstructed sample was injected on a C18 column using an isocratic elution. Subsequent MS/MS analysis was performed using an API 5500 mass detector (Sciex, Concord, Ontario, Canada) in positive electrospray mode.

Riociguat and M1 were extracted from plasma samples using basic liquid-liquid extraction. After evaporation of the organic solvent, the reconstructed sample was injected on a C18 column using an isocratic elution. Subsequent MS/MS analysis was performed using an API 4000 mass detector (Sciex, Concord, Ontario, Canada) in positive electrospray mode.

Macitentan and ACT-132577 were extracted from plasma samples using protein precipitation with acetonitrile/ethanol. The supernatant was injected on a C18 column using a gradient elution. Subsequent MS/MS analysis was performed using an API 5000 mass detector (Sciex, Concord, Ontario, Canada) in positive electrospray mode.

The quality of each analytical run was controlled by including quality control samples at low, medium and high concentrations. Their measured concentrations were used to determine intra- and inter-run precision and accuracy.

\subsection{Pharmacokinetic Assessments}

The plasma pharmacokinetic parameters of rosuvastatin, riociguat and M1 were derived by non-compartmental analysis (using Phoenix WinNonlin 6.4 [Certara, Princetion, NJ, USA]) of the plasma concentration-time profiles and included the following: the maximum plasma concentration of analyte $\left(C_{\max }\right)$, the area under the plasma concentrationtime curve (AUC) from zero (pre-dose) to time of the last measured concentration above the limit of quantification $\left(\mathrm{AUC}_{0-\mathrm{t}}\right)$, the AUC from zero to infinity $\left(\mathrm{AUC}_{0-\infty}\right)$, the time to reach maximum plasma concentration $\left(t_{\max }\right)$ and the terminal elimination half-life $\left(t_{1 / 2}\right)$.
The individual plasma concentrations of rosuvastatin, riociguat and M1 were used to directly obtain $C_{\max }$ and $t_{\max }$. $\mathrm{AUC}_{0-\mathrm{t}}$ was calculated according to the linear trapezoidal rule using the measured concentration-time values above the lower limit of quantification (LLOQ). $\mathrm{AUC}_{0-\infty}$ was calculated by combining $\mathrm{AUC}_{0-\mathrm{t}}$ and the extrapolated AUC (AUC extra). $\mathrm{AUC}_{\text {extra }}$ represents an extrapolated value obtained by $C_{\mathrm{t}} / \lambda_{z}$ where $C_{\mathrm{t}}$ is the last concentration above the LLOQ and $\lambda_{z}$ represents the terminal elimination rate constant determined by log-linear regression analysis of the measured plasma concentrations in the terminal elimination phase. The $t_{1 / 2}$ of rosuvastatin, riociguat and M1 was calculated as follows: $t_{1 / 2}=0.693 / \lambda_{z}$. The measured individual trough plasma concentrations of macitentan and ACT-132577 were used to directly obtain $C_{\text {trough }}$, which was used to determine the attainment of steady-state conditions.

\subsection{Safety Assessments}

Safety assessments were performed at several time points throughout the study and included the evaluation of treatment-emergent adverse events (TEAEs), serious adverse events (SAEs), 12-lead ECG variables, clinical laboratory parameters (biochemistry, hematology, serology and urinalysis), vital signs (blood pressure and pulse), body weight and physical examination findings. An end of study examination was performed 10-12 days after last study drug administration and a follow-up telephone call to record any TEAEs and SAEs was performed 30-32 days after last study drug administration. TEAEs and SAEs were coded according to the Medical Dictionary for Regulatory Activities (version 20.0).

\subsection{Statistical Analysis}

A formal sample size calculation was not performed; however, based on previous publications, a precision estimate was calculated with an assumed coefficient of variation within subjects of $34 \%$ for $C_{\max }$ rosuvastatin, $21 \%$ for AUC 
rosuvastatin, $20 \%$ for $C_{\max }$ riociguat and $20 \%$ for AUC riociguat $[23,24]$. It was estimated that, with a sample size of 16 evaluable subjects in each study, the lower and upper bounds of the $90 \%$ confidence interval (CI) for the geometric mean ratio would be approximately 0.81 and 1.23 for $C_{\max }$ rosuvastatin, 0.88 and 1.14 for $\mathrm{AUC}_{0-\infty}$ rosuvastatin, 0.88 and 1.13 for $C_{\max }$ riociguat and, 0.88 and 1.13 for $\mathrm{AUC}_{0-\infty}$ riociguat if the estimated ratio was one. It was planned to recruit 20 subjects in each study to ensure 16 subjects with evaluable pharmacokinetic parameters were available for the analysis.

Both studies had two analysis sets: the all-treated set, which comprised all enrolled subjects who received at least one dose of the study drug, and the per-protocol analysis set, which comprised all subjects in the all-treated set with no major protocol deviations that could affect the evaluation of pharmacokinetic endpoints.

Statistical Analysis System $\left(\mathrm{SAS}^{\circledR}\right)$ software, version 9.4 (SAS Institute, Cary, NC, US) was used for the statistical analysis and the reporting of clinical and pharmacokinetics data. For the concentration-time profile plots of the pharmacokinetics data, WinNonlin version 6.4 or higher (Certara, Princeton, NJ, US) was used. Descriptive statistics were used to summarize the plasma concentrations per time point and the pharmacokinetic parameters of rosuvastatin, riociguat and $\mathrm{M} 1$ and $C_{\text {trough }}$ of macitentan and ACT-132577. The effect of macitentan on $C_{\max }, \mathrm{AUC}_{0-\mathrm{t}}$, $\mathrm{AUC}_{0-\infty}$ and $t_{1 / 2}$ of rosuvastatin, riociguat and M1 was determined using the geometric mean ratio and the $90 \% \mathrm{CI}$ of the test treatment versus the reference treatment (i.e. rosuvastatin and macitentan:rosuvastatin alone and riociguat and macitentan:riociguat alone). The geometric mean ratios and their $90 \%$ CIs were calculated from the corresponding back log-transformed contrasts of the mixed-effect models for $C_{\text {max }}, \mathrm{AUC}_{0-t}, \mathrm{AUC}_{0-\infty}$ and $t_{1 / 2}$ of rosuvastatin, riociguat or M1. A $90 \%$ CI of $0.80-1.25$ indicated bioequivalence. Differences between treatments for $t_{\max }$ were determined using the Wilcoxon signed rank test.

\section{Results}

\subsection{Study Population}

The rosuvastatin and riociguat studies enrolled 20 healthy male subjects each, all of whom received the study drugs and were included in the all-treated analysis set. In the rosuvastatin study, 18 subjects were included in the per-protocol analysis set; one subject discontinued the study due to TEAEs (see safety results) and one subject withdrew consent. In the riociguat study, all 20 subjects were included in the per-protocol analysis set. Demographics and baseline characteristics are summarized in Table 2.

\subsection{Bioanalysis}

Intra-run precision and accuracy were evaluated only in case the analytical batch contained a number of quality control samples at each concentration $\geq 3$ (for macitentan and ACT-132577 only). Intra-run precision was $\leq 8.1 \%$ for ACT-064992 and $\leq 6.9 \%$ for ACT-132577. Intra-run accuracy ranged from -9.8 to $7.4 \%$ for ACT-064992 and from -11.9 to $6.2 \%$ for ACT- 132577 .

Inter-run precision was $\leq 6.1 \%$ for rosuvastatin, $\leq 5.7 \%$ for riociguat, $\leq 5.0 \%$ for $\mathrm{M} 1, \leq 7.7 \%$ for macitentan and $\leq 6.2 \%$ for ACT-132577. Inter-run accuracy ranged from -9.3 to $4.0 \%$ for rosuvastatin, from -6.5 to $1.1 \%$ for riociguat, from -3.0 to $2.8 \%$ for M1, from -3.9 to $5.9 \%$ for macitentan and from -4.1 to $3.6 \%$ for ACT-132577.

\subsection{Pharmacokinetics}

\subsubsection{Effect of Macitentan on Rosuvastatin Pharmacokinetics}

The concentration-time profiles of rosuvastatin following administration of rosuvastatin $10 \mathrm{mg}$ alone or with macitentan $10 \mathrm{mg}$ were similar (Fig. 2a). The pharmacokinetic parameters of rosuvastatin in the absence and presence of macitentan are summarized in Table 3. Concomitant dosing of macitentan $10 \mathrm{mg}$ did not cause any notable difference in the rate and extent of systemic exposure $\left(C_{\max }, \mathrm{AUC}_{0-\mathrm{t}}\right.$ and $\mathrm{AUC}_{0-\infty}$ ) or $t_{1 / 2}$ of rosuvastatin when compared to treatment with rosuvastatin alone. The geometric mean ratios and their $90 \%$ CIs for rosuvastatin pharmacokinetic parameters were all within the $0.80-1.25$ limits (Table 3 ). There was no statistical difference between the two treatments for $t_{\max }$ of rosuvastatin.

Table 2 Demographics and baseline characteristics of the healthy male subjects enrolled in the rosuvastatin and riociguat studies, alltreated sets

\begin{tabular}{lll}
\hline Characteristic & $\begin{array}{l}\text { Rosuvastatin study } \\
N=20\end{array}$ & $\begin{array}{l}\text { Riociguat study } \\
N=20\end{array}$ \\
\hline $\begin{array}{l}\text { Age, years, mean (SD) } \\
\text { Race, } n(\%)\end{array}$ & $41.2(12.5)$ & $35.8(7.7)$ \\
$\quad$ White & $19(95)$ & $19(95)$ \\
American Indian or Alaska & $1(5)$ & 0 \\
$\quad$ Native & & $1(5)$ \\
Asian & 0 & $82.4(10.4)$ \\
Weight, kg, mean (SD) & $81.8(9.1)$ & $180.0(7.8)$ \\
Height, cm, mean (SD) & $182.8(6.3)$ & $25.4(2.7)$ \\
BMI, kg m ${ }^{2}$, mean (SD) & $24.8(2.7)$ & \\
\hline
\end{tabular}

$B M I$ body mass index, $S D$ standard deviation 


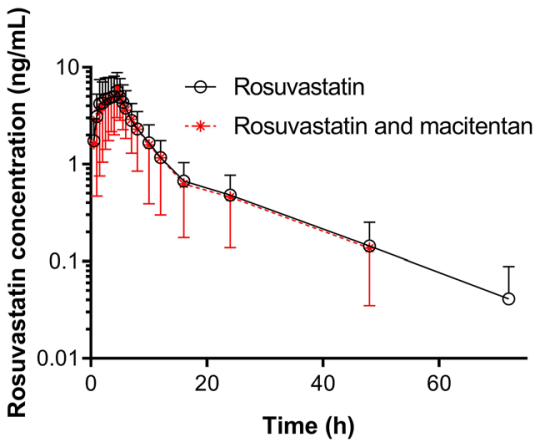

(a)

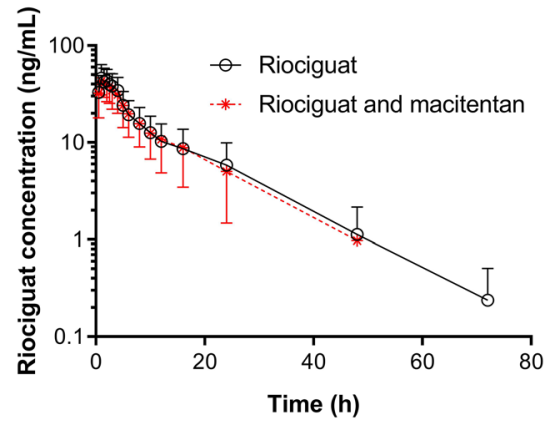

(b)

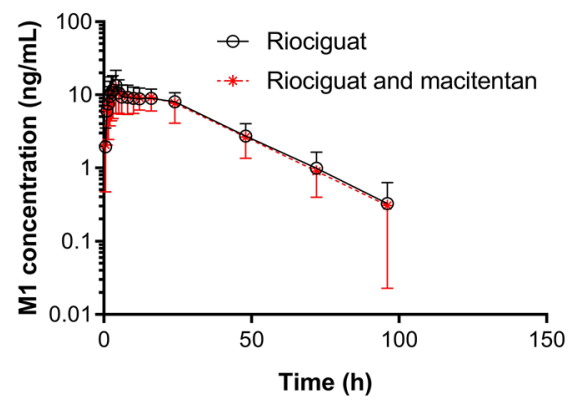

(c)
Fig. 2 a Mean and standard deviation (SD) plasma concentrationtime profiles of rosuvastatin following treatment with $10 \mathrm{mg}$ rosuvastatin alone and $10 \mathrm{mg}$ rosuvastatin with $10 \mathrm{mg}$ macitentan, per-protocol set $(N=18)$. b Mean (SD) plasma concentration-time profiles of riociguat following treatment with $1 \mathrm{mg}$ riociguat alone and $1 \mathrm{mg}$ riociguat with $10 \mathrm{mg}$ macitentan, per-protocol set $(N=20)$. c Mean (SD) plasma concentration-time profiles of riociguat's metabolite, $\mathrm{M} 1$, following treatment with $1 \mathrm{mg}$ riociguat alone and $1 \mathrm{mg}$ riociguat with $10 \mathrm{mg}$ macitentan, per-protocol set $(N=20)$

Table 3 Pharmacokinetic parameters of rosuvastatin following administration of $10 \mathrm{mg}$ rosuvastatin alone and rosuvastatin $10 \mathrm{mg}$ with maciten$\tan 10 \mathrm{mg}$, per-protocol set $(N=18)$

\begin{tabular}{|c|c|c|c|}
\hline \multirow{2}{*}{$\begin{array}{l}\text { Rosuvastatin pharmacokinetic param- } \\
\text { eter }\end{array}$} & \multicolumn{2}{|c|}{ Geometric mean $(95 \% \mathrm{CI})^{\mathrm{a}}$} & \multirow{2}{*}{$\begin{array}{l}\text { Geometric mean } \\
\text { ratio }(90 \% \mathrm{CI})^{\mathrm{b}}\end{array}$} \\
\hline & $10 \mathrm{mg}$ rosuvastatin & $10 \mathrm{mg}$ rosuvastatin $+10 \mathrm{mg}$ macitentan & \\
\hline$C_{\max }(\mathrm{ng} / \mathrm{mL})$ & $5.14(3.81,6.93)$ & $5.58(4.25,7.31)$ & $1.09(0.98,1.21)$ \\
\hline $\mathrm{AUC}_{0-\mathrm{t}}(\mathrm{ng} \cdot \mathrm{h} / \mathrm{mL})$ & $47.20(35.34,63.04)$ & $45.71(33.49,62.38)$ & $0.97(0.88,1.06)$ \\
\hline $\mathrm{AUC}_{0-\infty}(\mathrm{ng} \cdot \mathrm{h} / \mathrm{mL})$ & $54.27^{\mathrm{c}}(41.87,70.35)$ & $50.81^{\mathrm{d}}(37.66,68.54)$ & $0.96^{\mathrm{e}}(0.85,1.08)$ \\
\hline$t_{1 / 2}(\mathrm{~h})$ & $14.83^{\mathrm{c}}(12.73,17.28)$ & $16.17^{\mathrm{c}}(13.16,19.87)$ & $1.14^{\mathrm{d}}(0.98,1.32)$ \\
\hline$t_{\max }(\mathrm{h})$ & $4.50(1.5-5.5)$ & $4.50(3.5-5.5)$ & $0.00(0.00,0.75)$ \\
\hline
\end{tabular}

$A U C_{(0-t)}$ area under the plasma concentration-time curve from zero (pre-dose) to time of the last measured concentration above the limit of quantification, $A U C_{(0-\infty)}$ area under the plasma concentration-time curve from zero to infinity, $C I$ confidence interval, $C_{\text {max }}$ maximum plasma concentration, $h$ hour, $t_{1 / 2}$ terminal elimination half-life, $t_{\max }$ time to reach maximum plasma concentration

${ }^{\mathrm{a}}$ For $t_{\mathrm{max}}$, median and range is shown

${ }^{\mathrm{b}}$ For $t_{\mathrm{max}}$, median difference and $90 \% \mathrm{CI}$ is shown

${ }^{\mathrm{c}} n=16$

$\mathrm{d}_{n=15}$

$\mathrm{e}_{n=14}$

\subsubsection{Effect of Macitentan on Riociguat and M1 Pharmacokinetics}

The concentration-time profiles of riociguat and M1 following administration of riociguat $1 \mathrm{mg}$ alone or with maciten$\tan 10 \mathrm{mg}$ were similar (Fig. 2b, c). The pharmacokinetic parameters of riociguat and $\mathrm{M} 1$ in the absence and presence of macitentan are summarized in Tables 4 and 5. Concomitant dosing of macitentan $10 \mathrm{mg}$ resulted in similar systemic exposure $\left(C_{\max }, \mathrm{AUC}_{0-\mathrm{t}}\right.$ and $\left.\mathrm{AUC}_{0-\infty}\right)$ and $t_{1 / 2}$ of riociguat and $\mathrm{M} 1$ when compared to treatment with riociguat alone. The geometric mean ratios and their $90 \%$ CIs for riociguat pharmacokinetic parameters were all within the 0.80-1.25 limits. There was no statistical difference between the two treatments for $t_{\max }$ of riociguat and $t_{\max }$ of M1.

\subsubsection{Steady-state Plasma Concentrations of Macitentan and ACT-132577}

Steady-state conditions for macitentan and ACT-132577 were reached before rosuvastatin or riociguat administration on Day 10. Rosuvastatin or riociguat did not have an effect on the steady-state concentrations of macitentan and ACT-132577 (Fig. 3a, b).

In the riociguat study, one subject had extremely low concentrations of macitentan and ACT-132577. A careful review of the demographic variables and the clinical and 
Table 4 Pharmacokinetic parameters of riociguat following administration of $1 \mathrm{mg}$ riociguat alone and riociguat $1 \mathrm{mg}$ with macitentan $10 \mathrm{mg}$, per-protocol set $(N=20)$

\begin{tabular}{|c|c|c|c|}
\hline \multirow{2}{*}{$\begin{array}{l}\text { Riociguat pharmacokinetic } \\
\text { parameter }\end{array}$} & \multicolumn{2}{|l|}{ Geometric mean $(95 \% \mathrm{CI})^{\mathrm{a}}$} & \multirow{2}{*}{$\begin{array}{l}\text { Geometric mean } \\
\text { ratio }(90 \% \mathrm{CI})^{\mathrm{b}}\end{array}$} \\
\hline & $1 \mathrm{mg}$ riociguat & $1 \mathrm{mg}$ riociguat $+10 \mathrm{mg}$ macitentan & \\
\hline$C_{\max }(\mathrm{ng} / \mathrm{mL})$ & $47.97(41.61,55.29)$ & $45.96(40.02,52.78)$ & $0.96(0.90,1.02)$ \\
\hline $\mathrm{AUC}_{0-\mathrm{t}}(\mathrm{ng} \bullet \mathrm{h} / \mathrm{mL})$ & $406.26(296.39,556.86)$ & $386.32(286.34,521.22)$ & $0.95(0.83,1.09)$ \\
\hline $\mathrm{AUC}_{0-\infty}(\mathrm{ng} \bullet \mathrm{h} / \mathrm{mL})$ & $411.16(300.48,562.60)$ & $393.25(292.23,529.18)$ & $0.96(0.84,1.09)$ \\
\hline$t_{1 / 2}(\mathrm{~h})$ & $8.22(6.67,10.12)$ & $7.94(6.37,9.92)$ & $0.97(0.88,1.06)$ \\
\hline$t_{\max }(\mathrm{h})$ & $1.00(0.5-2.1)$ & $1.00(0.5-3.0)$ & $0.00(0.00,0.25)$ \\
\hline
\end{tabular}

$A U C_{(0-t)}$ area under the plasma concentration-time curve from zero (pre-dose) to time of the last measured concentration above the limit of quantification, $A U C_{(0-\infty)}$ area under the plasma concentration-time curve from zero to infinity, $C I$ confidence interval, $C_{\max }$ maximum plasma concentration, $h$ hour, $t_{1 / 2}$ terminal elimination half-life, $t_{\max }$ time to reach maximum plasma concentration

${ }^{\mathrm{a}}$ For $t_{\text {max }}$, median and range is shown

${ }^{\mathrm{b}}$ For $t_{\max }$, median difference and $90 \% \mathrm{CI}$ is shown

Table 5 Pharmacokinetic parameters of riociguat's metabolite, M1, following administration of riociguat $1 \mathrm{mg}$ alone and riociguat $1 \mathrm{mg}$ with macitentan $10 \mathrm{mg}$, per-protocol set $(N=20)$

\begin{tabular}{llll}
\hline M1 pharmacokinetic parameter & Geometric mean $(95 \% \mathrm{CI})^{\mathrm{a}}$ & & $\begin{array}{c}\text { Geometric mean } \\
\text { ratio }(90 \% \mathrm{CI})^{\mathrm{b}}\end{array}$ \\
\cline { 2 - 4 } & $1 \mathrm{mg}$ riociguat & $1 \mathrm{mg}$ riociguat +10 mg macitentan & $0.98(0.86,1.13)$ \\
\hline$C_{\max }(\mathrm{ng} / \mathrm{mL})$ & $12.10(9.28,15.77)$ & $11.91(9.45,15.00)$ & $0.98(0.91,1.06)$ \\
$\mathrm{AUC}_{0-\mathrm{t}}(\mathrm{ng} \cdot \mathrm{h} / \mathrm{mL})$ & $379.90(325.04,444.01)$ & $373.79(317.46,440.11)$ & $0.97(0.91,1.05)$ \\
$\mathrm{AUC}_{0-\infty}(\mathrm{ng} \cdot \mathrm{h} / \mathrm{mL})$ & $392.10(336.30,457.16)$ & $381.95(325.67,447.96)$ & $0.96(0.87,1.05)$ \\
$t_{1 / 2}(\mathrm{~h})$ & $15.63(14.12,17.30)$ & $14.94(13.28,16.80)$ & $0.50(-0.25,1.00)$ \\
$t_{\max }(\mathrm{h})$ & $4.00(3.0-24.0)$ & $5.00(2.5-23.9)$ & $(0.90)$ \\
\hline
\end{tabular}

$A U C_{(0-t)}$ area under the plasma concentration-time curve from zero (pre-dose) to time of the last measured concentration above the limit of quantification, $A U C_{(0-\infty)}$ area under the plasma concentration-time curve from zero to infinity, $C I$ confidence interval, $C_{\max }$ maximum plasma concentration, $h$ hour, $t_{1 / 2}$ terminal elimination half-life, $t_{\max }$ time to reach maximum plasma concentration

${ }^{\mathrm{a}}$ For $t_{\text {max }}$, median and range is shown

${ }^{\mathrm{b}}$ For $t_{\text {max }}$, median difference and $90 \% \mathrm{CI}$ is shown

bioanalytical procedures did not provide an explanation for the low levels. A sensitivity analysis of the pharmacokinetic parameters for riociguat and M1 was performed excluding this subject. Results of this analysis were similar to the results including all 20 subjects and the same conclusions were drawn.

\subsection{Safety}

There were no TEAEs of severe intensity, no deaths or other SAEs reported during the studies. In addition, there were no clinically significant changes from baseline in laboratory parameters, vital signs or ECG variables. In the rosuvastatin study, one subject discontinued due to the TEAEs toothache and tooth abscess, which were considered by the investigator to be not related to the study drugs. In the riociguat study, there were no TEAEs that lead to discontinuation of study drug.

In the rosuvastatin study, nine (45\%) subjects had at least one TEAE during the study. Headache was the most frequent TEAE, reported by three subjects treated with macitentan alone and four subjects treated with rosuvastatin and macitentan. In the riociguat study, $10(50 \%)$ subjects had at least one TEAE. Headache was also the most frequent TEAE in this study, reported by two subjects treated with riociguat alone, two subjects treated with macitentan alone and six subjects treated with riociguat and macitentan. In both studies, headache was the most frequently reported TEAE considered by the investigator to be related to the study drugs. The TEAE profiles in both studies were consistent with the known safety profiles of the study drugs. 


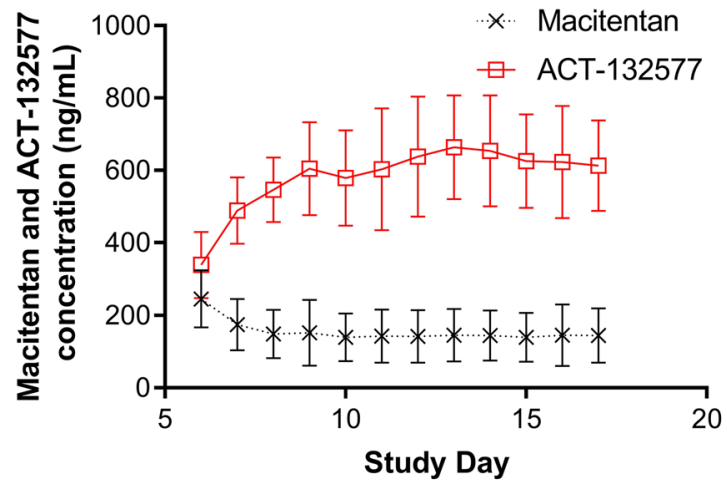

(a)

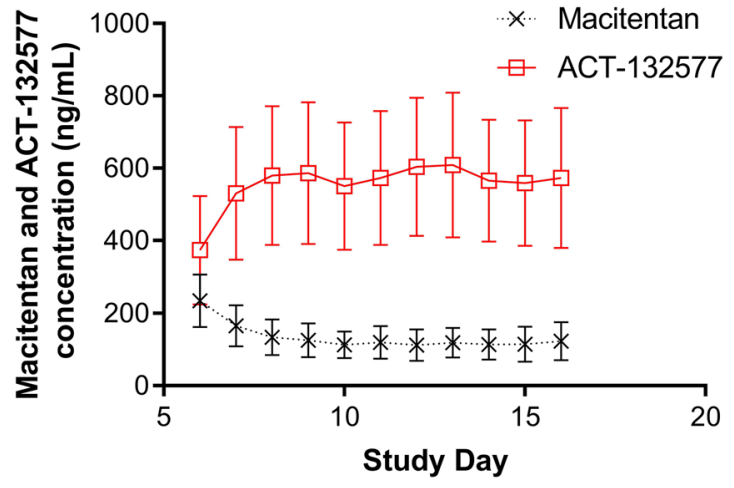

(b)

Fig. 3 Mean and standard deviation (SD) plasma concentration-time profiles of macitentan and its metabolite, ACT-132577, before and after administration of a rosuvastatin $(N=18)$ and $\mathbf{b}$ riociguat $(N=20)$ on Day 10 , per-protocol sets

\section{Discussion and Conclusion}

We conducted two studies to investigate the effect of concomitant administration of macitentan on the BCRP substrates, rosuvastatin and riociguat. Over $99 \%$ of macitentan and ACT-132577 binds to plasma proteins [12]. The resulting unbound plasma concentration of macitentan is not expected to lead to inhibition of BCRP-mediated transport in the liver or the kidney. However, the effect, if any, that macitentan has on intestinal BCRP has remained unknown until now. Our data showed that oral administration of macitentan $10 \mathrm{mg}$ had no effect on the pharmacokinetic profiles of orally administered rosuvastatin $10 \mathrm{mg}$ or riociguat $1 \mathrm{mg}$. The pharmacokinetic parameters of rosuvastatin $[25,26]$ and riociguat $[27,28]$ were consistent with those reported by other groups. In addition, we found that rosuvastatin or riociguat did not affect the steady-state concentrations of macitentan and its active metabolite, ACT-132577. Concomitant administration of macitentan with rosuvastatin or riociguat was well tolerated. There were no SAEs or severe TEAEs during the studies and the TEAE profiles were consistent with the known safety profiles of the drugs.

Our study design conformed to regulatory guidelines on the conduct of DDI studies [29, 30]. A starting dose of macitentan $30 \mathrm{mg}$ followed by $10 \mathrm{mg}$ per day for 5 days ensured rapid attainment of steady-state conditions before administration of rosuvastatin or riociguat. Continued administration of macitentan $10 \mathrm{mg}$ once-daily until Day 15 or Day 16 ensured the maintenance of steady-state conditions during the entire pharmacokinetic sampling period. The usual therapeutic dose of macitentan $10 \mathrm{mg}$ and the recommended start dose of rosuvastatin $10 \mathrm{mg}$ was chosen for the studies $[17,31]$. To avoid the risk of hypotension associated with riociguat, a dose of riociguat $1 \mathrm{mg}$ was selected [18]. The doses were in line with those used by other groups in similar DDI studies $[32,33]$.
One of the shortcomings of the studies was the small sample size, which limits the interpretation of safety data. However, in both studies, the observed within-subject variability for $C_{\max }$ and $\mathrm{AUC}_{0-\infty}$ was similar or smaller than assumed for the sample size calculation, indicating that the selected sample size was sufficient for the pharmacokinetic purposes of the studies. Another limitation of the study, which could affect the generalizability of the results to the patients with $\mathrm{PAH}$, is that subjects of Asian race were excluded from taking part in the rosuvastatin study because of the differences in the metabolism of statins in Asians compared with Caucasians [26].

Despite these limitations our studies show that there is no pharmacokinetic interaction between macitentan and the BCRP substrates, rosuvastatin and riociguat, in healthy subjects. Given that the pharmacokinetic profile of macitentan in healthy subjects is similar to that in PAH patients, the results presented here can be extrapolated to PAH [34]. Our results also suggest that macitentan can be used in combination with other BCRP substrates. These data will allow for informed decisions on future treatment combinations in patients with $\mathrm{PAH}$ and CTEPH.

In summary, the results from our studies show that at the approved clinical dose of macitentan $10 \mathrm{mg}$ has no effect on the pharmacokinetics of orally administered rosuvastatin or riociguat.

Acknowledgements The sponsor of both studies was Actelion Pharmaceuticals Ltd. Medical writing support was provided by Claire Kilmartin and Rachel Beeby from Trilogy Writing \& Consulting $\mathrm{GmbH}$, funded by Actelion Pharmaceuticals Ltd.

Author Contributions DC was responsible for the pharmacokinetic analysis and interpretation of data; SB was responsible for the study design; AS was responsible for the study implementation and had direct responsibility for patients; MS was responsible for the analysis and interpretation of safety information collected in the study; RS was responsible for the statistical analysis; GS was responsible for 
the pharmacokinetic analysis; JJPR was responsible for the interpretation of the data. All authors reviewed, contributed to and approved the manuscript.

Data Availability Statement The data sharing policy of the Sponsor is available at https://www.janssen.com/clinical-trials/transparency. As noted on this site, requests for access to the study data can be submitted through the Yale Open Data Access (YODA) Project site at http:// yoda.yale.edu.

\section{Compliance with Ethical Standards}

Ethical Approval All procedures performed in studies involving human participants were in accordance with the ethical standards of the institutional and/or national research committee and with the 1964 Helsinki declaration and its later amendments or comparable ethical standards.

Informed Consent Informed consent was obtained from all individual participants included in the study.

Funding The studies were funded by Actelion Pharmaceuticals Ltd.

Conflict of interest Dénes Csonka, Shirin Bruderer, Marianne Soergel and Juan Jose Perez Ruixo are employees of Actelion Pharmaceuticals Ltd. Armin Schultz is an employee of the clinical research organization Clinical Research Services Mannheim $\mathrm{GmbH}$, which carried out the studies reported. Radka Stepanova is an employee of the clinical research organization Aixial s.r.o, which performed the biostatistics for the studies reported. Giancarlo Sabattini is an employee of Idorsia Pharmaceuticals Ltd, which collaborated on the studies reported.

Open Access This article is distributed under the terms of the Creative Commons Attribution-NonCommercial 4.0 International License (http://creativecommons.org/licenses/by-nc/4.0/), which permits any noncommercial use, distribution, and reproduction in any medium, provided you give appropriate credit to the original author(s) and the source, provide a link to the Creative Commons license, and indicate if changes were made.

\section{References}

1. Lai YC, Potoka KC, Champion HC, et al. Pulmonary arterial hypertension: the clinical syndrome. Circ Res. 2014;115(1):115-30.

2. Giaid A, Yanagisawa M, Langleben D, et al. Expression of endothelin-1 in the lungs of patients with pulmonary hypertension. N Engl J Med. 1993;328(24):1732-9.

3. Rubens C, Ewert R, Halank M, et al. Big endothelin-1 and endothelin-1 plasma levels are correlated with the severity of primary pulmonary hypertension. Chest. 2001;120(5):1562-9.

4. Chester AH, Yacoub MH. The role of endothelin-1 in pulmonary arterial hypertension. Glob Cardiol Sci Pract. 2014;2014(2):62-78.

5. Arai $\mathrm{H}$, Hori $\mathrm{S}$, Aramori I, et al. Cloning and expression of a cDNA encoding an endothelin receptor. Nature. 1990;348(6303):730-2.

6. Sakurai T, Yanagisawa M, Takuwa Y, et al. Cloning of a cDNA encoding a non-isopeptide-selective subtype of the endothelin receptor. Nature. 1990;348(6303):732-5.

7. Lajoie AC, Bonnet S, Provencher S. Combination therapy in pulmonary arterial hypertension: recent accomplishments and future challenges. Pulm Circ. 2017;7(2):312-25.
8. McGoon MD, Miller DP. REVEAL: a contemporary US pulmonary arterial hypertension registry. Eur Respir Rev. 2012;21(123):8-18.

9. Monaco TJ, Davila CD. Safety, efficacy, and clinical utility of macitentan in the treatment of pulmonary arterial hypertension. Drug Des Dev Ther. 2016;10:1675-82.

10. Gatfield J, Mueller Grandjean C, Sasse T, et al. Slow receptor dissociation kinetics differentiate macitentan from other endothelin receptor antagonists in pulmonary arterial smooth muscle cells. PLoS One. 2012;7(10):e47662.

11. Sidharta PN, Treiber A, Dingemanse J. Clinical pharmacokinetics and pharmacodynamics of the endothelian receptor antagonist macitentan. Clin Pharmacokinet. 2015;54:457-71.

12. Summary of product characteristics. Opsumit ${ }^{\circledR}$ (macitentan) 10 mg tablets, for oral use. London: Actelion Registration, Ltd.; 2018.

13. Elsby R, Hilgendorf C, Fenner K. Understanding the critical disposition pathways of statins to assess drug-drug interaction risk during drug development: it's not just about OATP1B1. Clin Pharmacol Ther. 2012;92(5):584-98.

14. Frey R, Becker C, Saleh S, et al. Clinical pharmacokinetic and pharmacodynamic profile of riociguat. Clin Pharmacokinet. 2018;57(6):647-61.

15. Khaybullina D, Patel A, Zerilli T. Riociguat (adempas): a novel agent for the treatment of pulmonary arterial hypertension and chronic thromboembolic pulmonary hypertension. $\mathrm{P} \mathrm{T}$. 2014;39(11):749-58.

16. Liao JK. Safety and efficacy of statins in Asians. Am J Cardiol. 2007;99(3):410-4.

17. Crestor [10 mg film-coated tablets summary of product characteristics]. Bedfordshire, UK: AstraZeneca UK. 2018. https://www. medicines.org.uk/emc/product/7559/smpc. Accessed 04 Feb 2019.

18. Adempas [prescribing information]. Whippany, NJ: Bayer HealthCare Pharmaceuticals Inc. 2018. http://labeling.bayerhealthcare .com/html/products/pi/Adempas_PI.pdf. Accessed 04 Feb 2019.

19. Borkowski L, Steigerwald K. Validation of determination of rosuvastatin in plasma samples of subjects. ACC GmbH Analytical Clinical Concepts, Schöntalweg 9, 63849 Leidersbach, Germany. 2017.

20. Borkowski L, Steigerwald K. Validation of quantitative determination of the concentration of riociguat and its metabolite desmethyl-riociguat in plasma samples of subjects. ACC GmbH Analytical Clinical Concepts, Schöntalweg 9, 63849 Leidersbach, Germany. 2018

21. Zimmermann T. Validation of an analytical method for the determination of ACT-064992 and its metabolite ACT-132577 in human plasma samples by LC-MS/MS. Actelion Pharmaceuticals Ltd, Gewerbestrasse 16, 4123 Allschwil, Switzerland. 2014.

22. Steurer A. Supplementary validation of an analytical method for the determination of ACT-064992 and its metabolite ACT-132577 in human plasma samples by LC-MS/MS. Actelion Pharmaceuticals Ltd, Gewerbestrasse 16, 4123 Allschwil, Switzerland. 2016.

23. Martin P, Gillen M, Ritter J, et al. Effects of fostamatinib on the pharmacokinetics of oral contraceptive, warfarin, and the statins rosuvastatin and simvastatin: results from phase I clinical studies. Drugs R D. 2016;16(1):93-107.

24. Frey R, Reber M, Krätzschmar J, et al. Riociguat (BAY 63-2521) and aspirin: a randomized, pharmacodynamic, and pharmacokinetic interaction study. Pulm Circ. 2016;6(Suppl 1):S35-42.

25. Martin PD, Warwick MJ, Dane AL, et al. A double-blind, randomized, incomplete crossover trial to assess the dose proportionality of rosuvastatin in healthy volunteers. Clin Ther. 2003;25(8):2215-24.

26. Birmingham BK, Bujac SR, Elsby R, et al. Rosuvastatin pharmacokinetics and pharmacogenetics in Caucasian and Asian 
subjects residing in the United States. Eur J Clin Pharmacol. 2015;71(3):329-40.

27. Becker C, Frey R, Unger S, et al. Pharmacokinetic interaction of riociguat with ketoconazole, clarithromycin, and midazolam. Pulm Circ. 2016;6(Suppl 1):S49-57.

28. Saleh S, Frey R, Becker C, et al. Bioavailability, pharmacokinetics, and safety of riociguat given as an oral suspension or crushed tablet with or without food. Pulm Circ. 2016;6(Suppl 1):S66-74.

29. European Medicines Agency, Evaluation of Medicines for Human Use, CPMP. Guideline on the investigation of Drug Interactions. CPMP/EWP/560/95 Rev. 1. London: 2012.

30. US Food and Drug Administration, Center for Drug Evaluation and Research (CDER): Guidance for industry. Drug Interaction Studies-Study design, data analysis, implications for dosing, and labeling recommendations, Rockville MD: February 2012.
31. OPSUMIT [prescribing information]. South San Francisco, CA: Actelion Pharmaceuticals US, Inc. 2018. https://opsumit.com/ opsumit-prescribing-information.pdf. Accessed 04 Feb 2019.

32. Polli JW, Hussey E, Bush M, et al. Evaluation of drug interactions of GSK1292263 (a GPR119 agonist) with statins: from in vitro data to clinical study design. Xenobiotica. 2013;43(6):498-508.

33. Becker C, Frey R, Hesse C, et al. Absorption of riociguat (BAY 63-2521): bioavailability, food effects, and dose proportionality. Pulm Circ. 2016;6(Suppl 1):S27-34.

34. Issac M, Dingemanse J, Sidharta PN. Pharmacokinetics of macitentan in patients with pulmonary arterial hypertension and comparison with healthy subjects. J Clin Pharmacol. 2017;57(8):997-1004. 